\title{
Personalized individual semantics based approach to MAGDM with the linguistic preference information on alternatives
}

\author{
Yuexuan Wang ${ }^{1}$, Yucheng Dong ${ }^{1}$, Hengjie Zhang ${ }^{2, *}$, Yuan Gao ${ }^{1}$ \\ ${ }^{I}$ Business School, Sichuan University, Chengdu 610065, China \\ ${ }^{2}$ Business School, Hohai University, Nanjing 211100, China \\ E-mails: yxwang@stu.scu.edu.cn (Yuexuan Wang) \\ ycdong@scu.edu.cn (Yucheng Dong) \\ hengjiezhang@hhu.edu.cn (Hengjie Zhang) \\ gaoyuan1984@scu.edu.cn (Yuan Gao)
}

Received 11 June 2017

Accepted 5 January 2018

\begin{abstract}
Personalized individual semantics (PIS) exist widely in our daily life, and it means that different people have different understandings regarding the same word. In decision making, decision makers are accustomed to express their preferences using a linguistic way, and it is naturally that the PIS will influence the decision result in the linguistic decision making. In this paper, we propose a PIS-based MAGDM framework for multiple attribute group decision making (MAGDM) problems with the linguistic preference information on alternatives. In the novel framework, a two-stage based optimization model is constructed to deal with PIS by minimizing the deviation between objective preference information (i.e., multiple attribute decision matrix) and subjective preference information (i.e., linguistic preference relations over alternatives), and this optimization model is then transformed into a linear programming model that can be easily solved. Based on this, decision makers' linguistic preference information can be transformed into numerical preference information for implementing the computation process. By fusing objective and subjective preference information, the collective solution of MAGDM problem can be obtained. The numerical and simulation experiments are conducted to verify the effectiveness of the proposal.
\end{abstract}

Keywords: Computing with words, multiple attribute group decision making (MAGDM), personalized individual semantics (PIS), numerical scale

\section{Introduction}

Multiple attribute decision making (MADM) refers to the problem of ranking alternatives from best to worst based on the multiple attribute decision matrix associated with multiple alternatives and attributes [7, 19]. The MADM has been widely used in many areas, such as economics, management, engineering and so on $[4,41]$. As the decision-making environment becomes

\footnotetext{
* Corresponding author
}

more and more complicated, it is hard for a single decision maker (DM) to take account all aspects of a decision making problem. In some situations, MADM problem needs to be solved by a group of DMs. Therefore, multiple attribute group decision making (MAGDM) models have been proposed and used to deal with the complex situations of decision making problems. In general, solving MAGDM problems includes the following two steps: (1) aggregating the individual multiple attribute decision matrices into a collective multiple attribute decision matrix; 
obtaining the ranking of alternatives from collective multiple attribute decision matrix. The research on MAGDM has made a great progress, and the more information can be found in [11, 19, 42].

In the classical MAGDM problems, decision information only includes multiple attribute decision matrices that provided by a group of DMs. However, in some MAGDM problems, DMs will give preference information on alternatives besides multiple attribute decision matrices. This type of MAGDM problem is called MAGDM with preference information [20, 26]. Clearly, there are two kinds of preference information in MAGDM problem with preference information on alternatives: objective preference information (i.e., multiple attribute decision matrix) and subjective preference information (i.e., preference relations on alternatives). In the extant literature, Fan et al. [12] investigated MADM problem with fuzzy preference relation on alternatives. Moreover, Wang and Parkan [38] proposed some approaches to MADM with preference information, in which the attributes weights are incompletely known. Recently, Kadziński and Tervonen investigated MAGDM with additive value models and preference information on alternatives [20].

To our knowledge, most of the existing studies on MAGDM with preference information assumed that the DMs provide their preference information over alternatives using a numerical way $[12,26,38]$. However, in practice, some DMs may feel more comfortable to use a linguistic way to provide their preference information over alternatives instead of using a numerical way. In recent years, linguistic decision making has become a hot research topic in the decision area, and a lot of linguistic decision making models have been reported [5, 6, 9, 25, 31, 33, 34, 40, 47, 48]. When DMs use a linguistic way to provide preference information, it is natural that words mean different things for different DMs. In other words, DMs have personalized individual semantics (PIS) in linguistic decision making (e.g., $[1,27,28])$. PIS exist widely in our daily life. For example, during an interview, two interviewers both give comments "good" to an interviewee. However, the term "Good" may has different meanings, one interviewer may think this interviewee should score 80 points, while the other interviewer may think the interviewee should score 95 points, if using the hundred percentage point system. Therefore, PIS are important elements that cannot be ignored in linguistic decision making due to their influences on the final decision result. [21]. Recently, Li et al. [21] investigated the PIS in the group decision making with linguistic preference relations. The basic idea to deal with PIS presented in [21] is that if the consistency level of a linguistic preference relation is acceptable, then the consistency level of the numerical preference relation that converted from it should be as better as possible.

Surely, the PIS will also exist in MAGDM with linguistic preference information over alternatives. To our knowledge, there is no research that focused on this issue. To fill this gap, this paper proposes a PIS based framework for MAGDM problem with linguistic preference information on alternatives, which is inspired by the work of [21]. In the proposed framework, a two-stage based optimization model is proposed to yield the numerical scales of linguistic terms with PIS. In the first stage, an optimization model is designed to obtain the numerical scales of linguistic terms and the attributes weights by minimizing the deviation between objective (multiple attribute decision matrix) and subjective (linguistic preference relations over alternatives) preference information. However, the optimization model presented in first stage may have multiple solutions. To deal with this problem, the second-stage optimization model is presented to further optimize the solutions obtained from the first-stage optimization model. Both optimization models proposed in the first and second stages are transformed into linear programming models that can be easily solved. Based on this, the linguistic preference relations over alternatives are transformed into additive preference relations. Further, the collective solution can be obtained by fusing objective preference information (i.e., multiple attribute decision matrix) and subjective preference information (i.e., linguistic preference relations over alternatives).

Although the investigation of the PIS in our paper is inspired by the work of [21], the approaches to deal with PIS between them are clearly different. The approach to deal with PIS in [21] is based on consistency-driven methodology, while in our proposal, we address PIS by minimizing the deviation between objective preference information (i.e., multiple attribute decision matrix) and subjective preference information (i.e., linguistic preference relations over alternatives). 
The rest of this paper is organized as follows. Section 2 introduces the 2-tuple linguistic model, numerical scale model and the MAGDM with numerical preference information on alternatives. In Section 3, a PIS based decision framework for MAGDM with linguistic preference on alternatives is proposed. The two-stage based optimization model is developed to support our framework, and a numerical example is used to show the applications of the proposed approach in Section 4. In Section 5, the numerical and simulation experiments are designed to verify the effectiveness of the proposal. Finally, concluding remarks are made in Section 6.

\section{Preliminaries}

This section provides some basic knowledge about the 2-tuple linguistic model, the numerical scale model, and the MAGDM problem with preference information on alternatives.

\subsection{The 2-tuple linguistic model}

This section introduces the 2-tuple linguistic model, which is the basis of numerical scale.

Zadeh [43] introduced the concept of linguistic variable as "a variable whose values are not numbers but words or sentences in a natural or artificial language". A linguistic value is less precise than a number but it is closer to human cognitive processes used to solve successfully problems dealing with uncertainty. Let $S=\left\{s_{i} \mid i=0,1,2, \ldots, g\right\}$ be a linguistic term set with odd cardinality. The term $s_{i}$ represents a possible value for a linguistic variable, and it is required that the linguistic term set should satisfy the following characteristics $[17,43]$ :

(1) The set is ordered: $s_{i}>s_{j}$ if and only if $i>j$;

(2) There is a negation operator: $\operatorname{Neg}\left(s_{i}\right)=s_{j}$ such that $j=g-i$.

The number of linguistic terms in the set $S$ is called the cardinality of $S$. If the semantics of the elements in the linguistic term set are given by fuzzy numbers [36] (defined in the [0,1] interval), then the midterm represents an assessment of "approximately $0.5 \%$

Definition 1. [17]: Let $\beta \in[0, g]$ be a number in the granularity interval of the linguistic term set
$S=\left\{s_{i} \mid i=0,1,2, \ldots, g\right\}$, and let $i=\operatorname{round}(\beta)$ and $\alpha=\beta-i$ be two values such that $i \in[0, g]$ and $\alpha \in[-0.5,0.5)$. Then, $\alpha$ is called a symbolic translation, with round being the usual rounding operation.

The 2-tuple linguistic representation model, presented in Herrera and Martínez [17] represents the linguistic information by a 2-tuple $\left(s_{i}, \alpha\right) \in \bar{S}=$ $S \times[-0.5,0.5)$, where $s_{i} \in S$ and $\alpha \in[-0.5,0.5)$.

Definition 2. $[17,24]$ Let $S=\left\{s_{i} \mid i=0,1,2, \ldots, g\right\}$ be a linguistic term set and $\beta \in[0, g)$ be a value representing the result of a symbolic aggregation operation. The 2-tuple that expresses the equivalent information to $\beta$ is then obtained as:

$\Delta:[0, g] \rightarrow S \times[-0.5,0.5)$,

where

$$
\Delta(\beta)=\left(s_{i}, \alpha\right), \text { with }\left\{\begin{array}{l}
s_{i}, i=\operatorname{round}(\beta) \\
\alpha=\beta-i, \alpha \in[-0.5,0.5)
\end{array}\right.
$$

Function $\Delta$, it is a one to one mapping whose inverse function $\Delta^{-1}: \bar{S} \rightarrow[0, g]$ is defined as $\Delta^{-1}\left(s_{i}, \alpha\right)=i+\alpha$. When $\alpha=0, \Delta(\beta)$ is then called a simple term.

Definition 3. [8] The matrix $L=\left(l_{i j}\right)_{n \times n}$, where $l_{i j} \in S$, is called a linguistic preference relation. The matrix $L=\left(l_{i j}\right)_{n \times n}$, where $l_{i j} \in \bar{S}$, is called a 2-tuple linguistic preference relation. If $l_{i j}=\operatorname{Neg}\left(l_{j i}\right)$ for $i, j=1,2, \ldots, n$, then $L$ is considered reciprocal.

In decision making with preference relation(s), the transitivity is an important concept. Let $s_{\alpha}\left(\alpha=\frac{g}{2}\right)$ is the midterm of linguistic term set S. Several transitive properties of linguistic preference relations are described as follows:

(1) Intransitivity: $l_{i j}>s_{\alpha}, \quad l_{j k}>s_{\alpha} \Rightarrow l_{k i}>s_{\alpha}$ for $i, j, k \in\{1,2, \ldots, n\}$

(2) Weak stochastic transitivity: $l_{i j} \geq s_{\alpha}, l_{j k} \geq s_{\alpha}$ $\Rightarrow l_{i k} \geq s_{\alpha}$ for $i, j, k \in\{1,2, \ldots, n\}$

(3) Strong stochastic transitivity: $l_{i j} \geq s_{\alpha}, l_{j k} \geq s_{\alpha}$ $\Rightarrow l_{i k} \geq \max \left(l_{i j}, l_{j k}\right)$ for $i, j, k \in\{1,2, \ldots, n\}$

(4) Additive transitivity: $l_{i j}=l_{i k}-l_{j k}+s_{\alpha}$ for $i, j, k \in\{1,2, \ldots, n\}$ 
Clearly, the additive transitivity condition is stronger than strong stochastic transitivity, and the strong stochastic transitivity condition is stronger than weak stochastic transitivity. In general, we consider that the linguistic preference relation is of acceptable consistency if it satisfies a set of pre-established transitive properties. In this study, we assume that the consistency level of the linguistic preference relation is acceptable if it satisfies the weak stochastic transitivity.

\subsection{Numerical scale model}

The concept of the numerical scale was introduced in [9], which is used to transform linguistic preference information into numerical preference for implementing calculation process.

Definition 4. [9] Let $S=\left\{s_{i} \mid i=0,1,2, \ldots, g\right\}$ be a linguistic term set, and $\mathrm{R}$ be the set of real numbers. The function: NS: $S \rightarrow \mathrm{R}$ is defined as a numerical scale of $S$, and $N S\left(s_{i}\right)$ is called the numerical index of $s_{i}$.

Definition 5. [9] Let $\mathrm{S}, \bar{S}$ and NS be as before. The numerical scale $\overline{N S}$ on $\bar{S}$ for $\left(s_{i}, \alpha\right) \in \bar{S}$, is defined by

$\overline{N S}\left(\left(s_{i}, \alpha\right)\right)=\left\{\begin{array}{l}N S\left(s_{i}\right)+\alpha \times\left(N S\left(s_{i+1}\right)-N S\left(s_{i}\right)\right), \alpha \geq 0 \\ N S\left(s_{i}\right)+\alpha \times\left(N S\left(s_{i}\right)-N S\left(s_{i-1}\right)\right), \alpha<0\end{array}\right.$

\subsection{MAGDM problem with numerical preference information on alternatives}

The MAGDM problem with numerical preference information on alternatives is described as follows:

Let $X=\left\{x_{1}, x_{2}, \ldots, x_{n}\right\} \quad(n \geq 2)$ be a finite set of alternatives, where $x_{i}$ denotes the $i$ th alternative; $E=\left\{e_{1}, e_{2}, \ldots, e_{m}\right\}$ be a finite set of experts, where $e_{k},(k=1,2, \ldots, m) \quad$ denotes the $k$ th expert; $A=\left\{a_{1}, a_{2}, \ldots, a_{t}\right\} \quad(\mathrm{t} \geq 2) \quad$ be a finite set of attributes, where $a_{j}$ denotes the $j$ th attribute. Let $V=\left(v_{i j}\right)_{n \times t}$ be a multiple attribute decision matrix, where $v_{i j}$ is the preference value of the alternative $x_{i}$ with respect to the attribute $a_{j}$. Let $\omega=\left(\omega_{1}, \omega_{2}, \ldots, \omega_{t}\right)^{T}$ be the attributes weight vector, where $\omega_{j} \geq 0$ is the weight of attribute $a_{j}$ and $\sum_{j=1}^{m} \omega_{j}=1$. In some MAGDM problems, DMs will provide preference information on alternatives $X=\left\{x_{1}, x_{2}, \ldots, x_{n}\right\}$, and preference relations (e.g., multiplicative preference relations [2, 3], additive preference relations $[15,23,32]$, linguistic preference relations $[8,22,44])$ are the useful tools for experts to provide such preference information. The decision objective is to obtain the ranking of alternatives based on the multiple attribute decision matrix and the experts' preference information on alternatives.

In extant literature regarding the MAGDM with preference information on alternatives, additive preference relations (also called fuzzy preference relations) are widely used. In addition, different preference relations can be transformed into other formats (see [14]). In the following, we introduce the additive preference relations in detail.

Definition 6. $[15,32]$ A matrix $P=\left(p_{i j}\right)_{n \times n}$, where $p_{i j} \in[0,1]$ and $p_{i j}+p_{j i}=1$ for $i . j=1,2, \ldots, n$, is called an additive preference relation over alternatives $X=\left\{x_{1}, x_{2}, \ldots, x_{n}\right\}$. Specifically, $p_{i j}>0.5$ denotes that alternative $x_{i}$ is better than $x_{j} ; p_{i j}<0.5$ signifies that alternative $x_{j}$ is better than $x_{i}$; $p_{i j}=0.5$ represents the equally importance between $x_{i}$ and $x_{j}$.

Generally, ordinal and cardinal [3] consistency measures are two common types of consistency for a preference relation. The former is closely related to the transitivity of the corresponding preference relation, and the latter is a stronger concept because it not only implies the transitivity of preferences, but also the intensity of preference expressed by comparisons.

Similar to the transitive properties of linguistic preference relations, the transitive properties of additive preference relations are presented below:

(1) Intransitivity: $p_{i j}>0.5, \quad p_{j k}>0.5 \Rightarrow p_{k i}$ $>0.5$ for all $i, j, k \in\{1,2, \ldots, n\}$

(2) Weak stochastic transitivity: $p_{i j} \geq 0.5, p_{j k}$ $\geq 0.5 \Rightarrow p_{i k} \geq 0.5$ for all $i, j, k \in\{1,2, \ldots, n\}$

(3) Strong stochastic transitivity: $p_{i j} \geq 0.5, p_{j k}$ $\geq 0.5 \Rightarrow p_{i k} \geq \max \left(l_{i j}, l_{j k}\right)$ for all $i, j, k \in\{1,2, \ldots, n\}$

(4) Additive transitivity: $p_{i j}=p_{i k}-p_{j k}+0.5$ for all $i, j, k \in\{1,2, \ldots, n\}$.

Based on additive transitivity, the cardinal consistency measure for an additive preference relation is presented below. 
Definition 7. [16] The cardinal consistency index (CCI) of an additive preference relation, $P=\left(p_{i j}\right)_{n \times n}$, is presented below:

$C C I(P)=\frac{2}{3 n(n-1)(n-2)} \sum_{i, j, k=1 ; i \neq j \neq k, i \neq k}^{n}\left|p_{i j}+p_{j k}-p_{i k}-0.5\right|$

where $C C I(P) \in[0,1]$. If $C C I(P)=0$, then the preference relation $P=\left(p_{i j}\right)_{n \times n} \quad$ is completely consistent; otherwise, the smaller value of $\operatorname{CCI}(P)$, the more consistent of $P$.

\section{A PIS based MAGDM framework}

In this section, we propose a PIS based decision framework for MAGDM problem with linguistic preference information on alternatives.

\subsection{The description of the problem: MAGDM with linguistic preference information on alternatives}

As expounded in section 1, most of the studies assumed that the preference relations over alternatives in MAGDM were based on the numerical information. But sometimes in practical life, DMs may feel more comfortable to use a linguistic way to provide their preferences over alternatives. For implementing the calculation process, in linguistic decision making, the linguistic preference information is often transformed into the numerical preference information. When quantifying linguistic preference information, PIS are important elements that cannot be ignored due to their influences on the final decision. Based on above analysis, we present a new decision problem: MAGDM with linguistic preference information on alternatives, which is formally presented below.

Recall that $E=\left\{e_{1}, e_{2}, \ldots, e_{m}\right\}$ is a finite set of DMs; $X=\left\{x_{1}, x_{2}, \ldots, x_{n}\right\} \quad(n \geq 2)$ is a finite set of alternatives; $A=\left\{a_{1}, a_{2}, \ldots, a_{t}\right\} \quad(t \geq 2)$ is a finite set of attributes; $\omega=\left(\omega_{1}, \omega_{2}, \ldots, \omega_{t}\right)^{T}$ is the weight vector over attributes $A=\left\{a_{1}, a_{2}, \ldots, a_{t}\right\}$; and $V=\left(v_{i j}\right)_{n \times t}$ is a decision matrix. Let $\lambda=\left(\lambda_{1}, \lambda_{2}, \ldots, \lambda_{m}\right)^{T}$ be the weight vector of DMs, where $\lambda_{k} \geq 0$ denotes the weight of $\mathrm{DM} e_{k}$ and $\sum_{k=1}^{m} \lambda_{k}=1$. In decision making, DM $e_{k}$ uses linguistic term set $S=\left\{s_{0}, s_{1}, \ldots, s_{g}\right\}$ to provide their linguistic preference relations $L^{k}=\left(l_{i j}^{k}\right)_{n \times n}$ over alternatives $X=\left\{x_{1}, x_{2}, \ldots, x_{n}\right\}$, where $l_{i j}^{k} \in S$ denotes the preference degree of alternative $x_{i}$ over $x_{j}$.

In the decision making, DMs have PIS regarding their $L^{k}=\left(l_{i j}^{k}\right)_{n \times n}$. The problem in this paper is concerned with the ranking of alternatives or selection of the most desirable alternative(s) by fusing the objective preference information $V=\left(v_{i j}\right)_{n \times t}$ and the subjective preference information $L^{k}=\left(l_{i j}^{k}\right)_{n \times n}$.

\subsection{The proposed framework}

Here, we propose a new decision framework to handle MAGDM with linguistic preference relations on alternatives: PIS based MAGDM framework. This framework is described in Fig. 1, and two main steps are included in this framework: (1) transforming linguistic preference information into numerical preference information; (2) selection of the alternatives.

(1) Transforming linguistic preference information into numerical preference information

In this process, a consistency control process is utilized to improve the consensus level of linguistic preference relations. After that, a two-stage based optimization model is designed to yield the numerical scales with PIS by minimizing the deviation between objective (multiple attribute decision matrix) and subjective (transformed fuzzy preference relations over alternatives) preference information.

Note 1: The numerical preference relations are widely used in GDM, such as multiplicative preference relations [3], additive preference relations $[5,16]$, and interval additive preference relations [5]. Herrera-Viedma et al. [14] discussed the transformation function between different numerical preference relations. The use of numerical preference relations will not change the essence of the proposed MAGDM framework. Without loss of generality, we consider that the linguistic preference relations are converted into additive preference relations in this paper.

The details of this process are presented in Section 4.

(2) Selection of the alternatives

After getting the numerical scales $\left(N S^{k}\left(s_{i}\right)\right)$ with PIS from the above step, we can transform the linguistic preference relations $L^{k}=\left(l_{i j}^{k}\right)_{n \times n}$ into the numerical preference relations $P^{k}=\left(p_{i j}^{k}\right)_{n \times n}$. Next, the subjective preference vector $S P V^{(k)}=\left(S P V_{1}^{(k)}, \quad S P V_{2}^{(k)}, \ldots\right.$, 
$\left.S P V_{n}^{(k)}\right)^{T}$ can be obtained from $P^{K}$, where $S P V_{i}^{(k)}$ represents the subjective preference value of alternative $x_{i}$ and it can be obtained using Eq. (9). Further, we can get the collective subjective preference vector $S P V=$ $\left(S P V_{1}, S P V_{2}, \ldots, S P V_{n}\right)^{T}$ by aggregating $\left\{S P V_{i}^{(1)}\right.$, $\left.S P V_{i}^{(2)}, \ldots, S P V_{i}^{(m)}\right\} \quad(i=1,2, \ldots, n)$, where $S P V_{i}$ denotes the collective preference value of alternative $x_{i}$ and it can be calculated using the following way,

$$
S P V_{i}=\sum_{k=1}^{m} \lambda_{k} S P V_{i}^{(k)}, i=1,2, \ldots, n .
$$

Let $O P V=\left(O P V_{1}, O P V_{2}, \ldots, O P V_{n}\right)^{T}$ be the objective preference vector obtained from the multiple attribute decision matrix using Eq. (8), where $O P V_{i}$ represents the objective preference value from $x_{i}$.

After getting the subjective and objective preference vectors, the collective preference vector, $P V=\left(P V_{1}, P V_{2}, \ldots, P V_{n}\right)^{T}$, can be yielded, where the collective preference value of alternative $x_{i}$ is computed below:

$$
P V_{i}=\alpha O P V_{i}+(1-\alpha) S P V_{i}, i=1,2, \ldots, n,
$$

where $\alpha \in[0,1]$. Clearly, the larger $P V_{i}$ value denotes the better alternative $x_{i}$. Based on $P V=\left(P V_{1}, P V_{2}, \ldots, P V_{n}\right)^{T}$, the collective solution, i.e., the collective ranking of alternatives from best to the worst, can be obtained.

Discussion. By taking the PIS into account, the deviation between objective and subjective preference information in our proposal can be decreased compared with the traditional MAGDM framework, which will be shown in the comparison section. Meanwhile, we should be noted that this paper assumes that linguistic preference relations provided by DMs are complete. However, in some situations, it may be difficult for DMs to provide all elements in linguistic preference relations on alternatives, which results in the uses of incomplete linguistic preference relations [35, 37, 39, 48]. In future research, we plan to investigate the management of incomplete linguistic preference relations under the PIS based MAGDM framework.

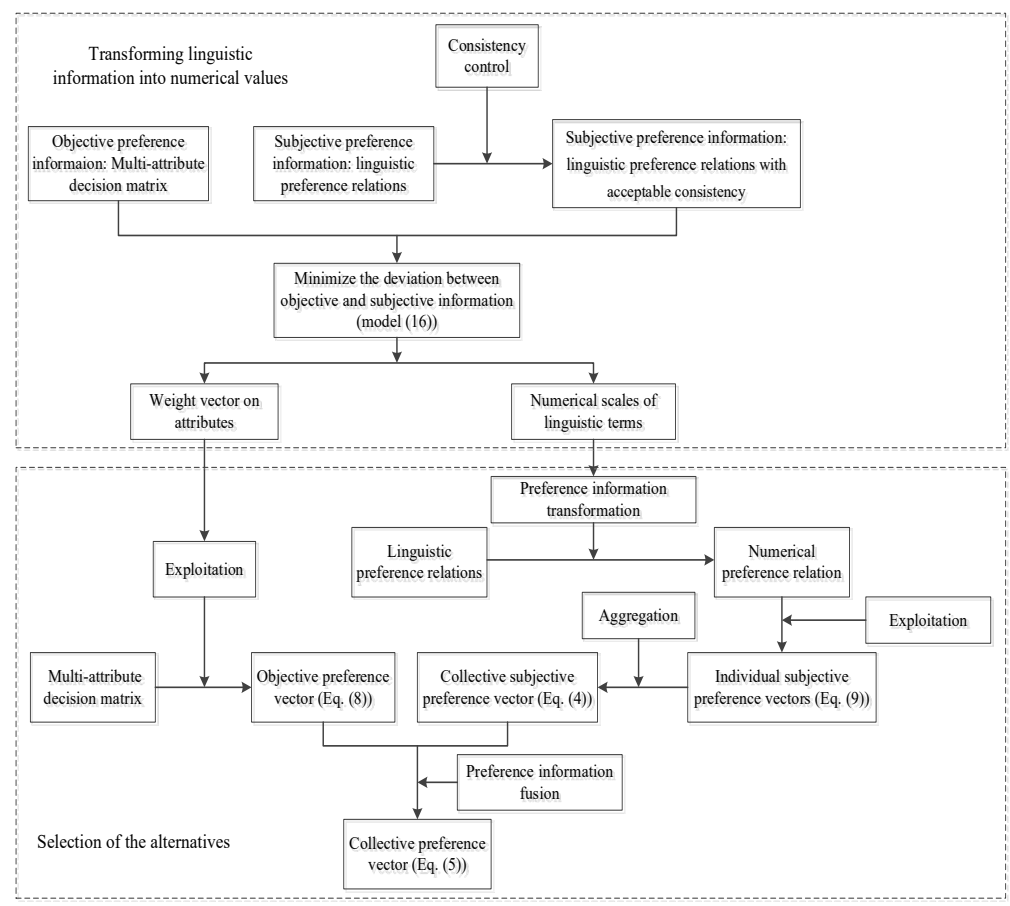

Fig. 1. The PIS based MAGDM framework

\section{Obtaining the individual numerical scales with PIS}

In this section, we propose a way to transform linguistic preference relations into numerical preference relations.
Specifically, Section 4.1 proposes a basic model for obtaining the individual numerical scales with PIS. Section 4.2 further optimizes the basic model presented in Section 4.1. In addition, a numerical example is provided to illustrate our method in Section 4.3. 


\subsection{Modeling}

Here, we propose a model to yield the individual numerical scales with PIS by minimizing the deviation between objective and subjective preference information. The model mainly includes three processes, which are presented below in detail.

(1) Obtaining objective preference vector from the multiple attribute decision matrix

In MAGDM problem, there are two categories of attributes: the benefit attributes and the cost attributes. Based on these two types of attributes, the multiple attribute decision matrix $V=\left(v_{i j}\right)_{n \times t}$ is transformed into normalized multiple attribute decision matrix $\bar{V}=\left(\overline{v_{i j}}\right)_{n \times t}$, where

$\overline{v_{i j}}=\frac{v_{i j}}{\sum_{i=1}^{n} v_{i j}}$ for benefit attribute $a_{j} \in A, j=1,2, \ldots, t(6)$ $\overline{v_{i j}}=\frac{1 / v_{i j}}{\sum_{i=1}^{n} 1 / v_{i j}}$ for cost attribute $a_{j} \in A, j=1,2, \ldots, t$

Let $O P V=\left(O P V_{1}, O P V_{2}, \ldots, O P V_{n}\right)^{T}$ be as above, where the objective preference value of alternative $x_{i}$ is exploited by [19]:

$$
O P V_{i}=\sum_{j=1}^{t} \overline{v_{i j}} \omega_{j}
$$

(2) Obtaining subjective preference vectors from the preference relations over alternatives

Let $P^{k}=\left(p_{i j}^{k}\right)_{n \times n}$ be the fuzzy preference relation associated with linguistic preference relation $L^{k}=\left(l_{i j}^{k}\right)_{n \times n}$, where $P^{k}=N S^{k}\left(l_{i j}^{k}\right)$. Let $S P V^{(k)}=$ $\left(S P V_{1}^{(k)}, S P V_{2}^{(k)}, \ldots, S P V_{n}^{(k)}\right)^{T}$ be as above, where $S P V_{i}^{(k)}$ is the subjective preference value of alternative $x_{i}$. Similar to the method to compute the preference vector of the interval additive preference relation [21], we adopt the following way to compute the subjective preference vector $S P V_{i}^{(k)}$,

$$
S P V_{i}^{(k)}=\frac{2}{n^{2}} N S^{k}\left(l_{i j}^{(k)}\right)=\frac{2}{n^{2}} \sum_{j=1}^{n} p_{i j}^{k}, i=1,2, \ldots, n ; k=1,2, \ldots, m .
$$

(3) Fusing the objective and subjective preference information

It is desirable that the deviation between the objective and subjective preference information is as small as possible. For DM $e^{k}$, the deviation is calculated by $\sum_{i=1}^{n}\left|O P V_{i}-S P V_{i}^{(k)}\right|$. For all DMs, the deviation is computed by $\sum_{k=1}^{m} \lambda_{k} \sum_{i=1}^{n}\left|O P V_{i}-S P V_{i}^{(k)}\right|$. Naturally, we hope $\sum_{k=1}^{m} \lambda_{k} \sum_{i=1}^{n}\left|O P V_{i}-S P V_{i}^{(k)}\right|$ is as small as possible, i.e.,

$$
\min \sum_{k=1}^{m} \lambda_{k} \sum_{i=1}^{n}\left|O P V_{i}-S P V_{i}^{(k)}\right|
$$

Further, Eq. (10) can be rewritten as follows:

$$
\begin{aligned}
& \min \sum_{k=1}^{m} \lambda_{k} \sum_{i=1}^{n}\left|\sum_{j=1}^{t} \omega_{j} \overline{v_{i j}}-\frac{2}{n^{2}} \sum_{j=1}^{n} p_{i j}^{k}\right| \\
= & \min \sum_{k=1}^{m} \lambda_{k} \sum_{i=1}^{n}\left|\sum_{j=1}^{t} \omega_{j} \overline{v_{i j}}-\frac{2}{n^{2}} \sum_{j=1}^{n} N S\left(l_{i j}^{k}\right)\right|
\end{aligned}
$$

Meanwhile, the numerical scales $N S^{k}\left(s_{i}\right)$, $k=1,2, \ldots, m$ should satisfy the following conditions:

(1) The numerical scale should be ordered, i.e.,

$$
N S^{k}\left(s_{i+1}\right)>N S^{k}\left(s_{i}\right), \quad i=0,1, \ldots, \frac{g}{2}, k=1,2, \ldots, m
$$

For controlling the discrimination degree between two consecutive numerical scales, we can take the following way:

$$
N S^{k}\left(s_{i+1}\right)-N S^{k}\left(s_{i}\right) \geq \gamma \quad k=1,2, \ldots, m ; i=0,1, \ldots, g-1
$$

where $\gamma \in[0,0.1]$ is a parameter. Without loss of generality, in this paper, we set $\gamma=0.05$.

(2) The numerical scale should be reciprocal, i.e.,

$$
N S^{k}\left(s_{i}\right)+N S^{k}\left(s_{g-i}\right)=1 \quad k=1,2, \ldots, m ; i=0,1,2, \ldots, \frac{g}{2}
$$

Moreover, for the flexibility of the numerical scales, we can set the range of $N S^{k}\left(s_{i}\right)$ as follows:

$$
\begin{cases}N S^{k}\left(s_{i}\right) \in[0,(i+\Delta) / g] & k=1,2, \ldots, m ; i=0,1, \ldots, \Delta-1 \\ \frac{(i-\Delta)}{g} \leq N S^{k}\left(s_{i}\right) \leq \frac{(i+\Delta)}{g} \quad k=1,2, \ldots, m ; i=\Delta, \Delta+1, \ldots, \frac{g}{2}\end{cases}
$$


where $\Delta \in\left[0, \frac{g}{2}\right]$, the bigger $\Delta$ value denotes the bigger range of $N S^{k}\left(s_{i}\right)$. Without loss of generality, in this paper, we set $\Delta=2$

In this study, we consider that the attribute weights are unknown. Thus, the objective preference vector derived from Eq. (8) is also unknown. Based on the above analyses (Eqs. (10)-(15)), we establish an optimization model to calculate the individual numerical scales with PIS and attribute weights:

$$
\begin{aligned}
& M=\min \sum_{k=1}^{m} \lambda_{k} \sum_{i=1}^{n}\left|\sum_{j=1}^{t} \omega_{j} \overline{v_{i j}}-\frac{2}{n^{2}} \sum_{j=1}^{n} p_{i j}^{k}\right| \\
& \text { s.t. } \begin{cases}p_{i j}^{k}=N S^{k}\left(l_{i j}^{k}\right) & k=1,2, \ldots, m ; i, j=1,2, \ldots, n \\
N S^{k}\left(s_{i}\right) \in[0,(i+\Delta) / g] & k=1,2, \ldots, m ; i=0,1, \ldots, \Delta-1 \\
\frac{(i-\Delta)}{g} \leq N S^{k}\left(s_{i}\right) \leq \frac{(i+\Delta)}{g} & k=1,2, \ldots, m ; i=\Delta, \Delta+1, \ldots, \frac{g}{2} \\
N S^{k}\left(s_{i+1}\right)-N S^{k}\left(s_{i}\right) \geq \gamma & k=1,2, \ldots, m ; i=0,1, \ldots, \frac{g}{2} \\
N S^{k}\left(s_{i}\right)+N S^{k}\left(s_{g-i}\right)=1 & k=1,2, \ldots, m ; i=0,1,2, \ldots, \frac{g}{2} \\
\sum_{j=1}^{t} \omega_{j}=1 & \\
\omega_{j} \geq 0 & j=1,2, \ldots, t .\end{cases}
\end{aligned}
$$

where $N S^{k}\left(s_{i}\right)(k=1,2, \ldots, m), \quad \omega_{j}(j=1,2, \ldots, t) \quad$ are the decision variables.

We denote this model as $P_{1}$. By analyzing model $P_{1}$, we present the following proposition.

Proposition 1. Let $N S^{(k, *)}\left(s_{i}\right)$ be the optimal solution to decision variable $N S^{(k)}\left(s_{i}\right)$ in model $P_{1}$. Let $P^{k}=\left(p_{i j}^{k}\right)_{n \times n}$ be the fuzzy preference relation associated with linguistic preference relation $L^{k}=\left(l_{i j}^{k}\right)_{n \times n}$, where $p_{i j}^{k}=N S^{(k, *)}\left(l_{i j}^{k}\right)$. Then, fuzzy preference relation $P^{k}=\left(p_{i j}^{k}\right)_{n \times n}$ satisfies weak stochastic transitivity.

Proof. The $L^{k}$ in model $P_{1}$ satisfies the weak stochastic transitivity, from Definition 3, we have $l_{i j}^{k} \geq s_{\alpha}, \quad l_{j r}^{k} \geq s_{\alpha} \Rightarrow l_{i r}^{k} \geq s_{\alpha}$ for $i, j, r \in\{1,2, \ldots, n\}$. Based on the properties of the numerical scale function, we have that: $p_{i j}^{k} \geq 0.5, \quad p_{j r}^{k} \geq 0.5 \Rightarrow p_{i r}^{k} \geq 0.5$ for $i, j, r \in\{1,2, \ldots, n\}$. According to the transitive properties introduced in Section 2.3, $P^{k}=\left(p_{i j}^{k}\right)_{n \times n}$ satisfies weak stochastic transitivity. This completes the proof of Proposition 1.
Clearly, model $P_{1}$ is a non-linear programming model. In the following, we transform it into a linear programming model that can be easily solved, which is shown in Theorem 1.

Theorem 1. Let $u_{i}{ }^{k} \geq 0$ be a variable, where $\sum_{j=1}^{t} \omega_{j} \overline{v_{i j}}-\frac{2}{n^{2}} \sum_{j=1}^{n} p_{i j}^{k} \leq u_{i}^{k}$ and $\quad-\left(\sum_{j=1}^{t} \omega_{j} \overline{v_{i j}}-\frac{2}{n^{2}} \sum_{j=1}^{n} p_{i j}^{k}\right) \leq u_{i}^{k}$. Then, model $P_{1}$ can be equivalently transformed into the following linear programming model:

$$
\begin{aligned}
& M=\min \sum_{k=1}^{m} \lambda_{k} \sum_{i=1}^{n} u_{i}^{k} \\
& \text { s.t. } \begin{cases}\sum_{j=1}^{t} \omega_{j} \overline{v_{i j}}-\frac{2}{n^{2}} \sum_{j=1}^{n} p_{i j}^{k} \leq u_{i}^{k} & k=1,2, \ldots, m ; i=1,2, \ldots, n \\
-\left(\sum_{j=1}^{t} \omega_{j} \overline{v_{i j}}-\frac{2}{n^{2}} \sum_{j=1}^{n} p_{i j}^{k}\right) \leq u_{i}^{k} & k=1,2, \ldots, m ; i=1,2, \ldots, n \\
p_{i j}^{k}=N S^{k}\left(l_{i j}\right) & k=1,2, \ldots, m ; i, j=1,2, \ldots, n \\
N S^{k}\left(s_{i}\right) \in[0,(i+\Delta) / g] & k=1,2, \ldots, m ; i=0,1, \ldots, \Delta-1 \\
g & k=1,2, \ldots, m ; i=\Delta, \Delta+1, \ldots, \frac{g}{2} \\
N S^{k}\left(s_{i}\right) \leq \frac{(i+\Delta)}{g} & k=1,2, \ldots, m ; i=0,1, \ldots, \frac{g}{2} \\
N S^{k}\left(s_{i}\right)+N S^{k}\left(s_{g-i}\right)=1 & k=1,2, \ldots, m ; i=0,1,2, \ldots, \frac{g}{2} \\
\sum_{j=1}^{t} \omega_{j}=1 & \\
\omega_{j} \geq 0 & j=1,2, \ldots, t\end{cases}
\end{aligned}
$$

Proof: In the model (17), constrains (2)-(4) guarantee that $\left|\sum_{j=1}^{t} \omega_{j} \overline{v_{i j}}-\frac{2}{n^{2}} \sum_{j=1}^{n} p_{i j}^{k}\right| \leq u_{i}^{k}$. According to the objective function of model (17), any feasible solution with $u_{i}^{k}>\left|\sum_{j=1}^{t} \omega_{j} \overline{v_{i j}}-\frac{2}{n^{2}} \sum_{j=1}^{n} p_{i j}^{k}\right|$ is not the optimal solution to model (17). Thus, constraints (2)-(4) guarantee that $u_{i}^{k}=\left|\sum_{j=1}^{t} \omega_{j} \overline{v_{i j}}-\frac{2}{n^{2}} \sum_{j=1}^{n} p_{i j}^{k}\right|$. So, model $P_{1}$ can be equivalently transformed into the model (17). This completes the proof of Theorem 1.

\subsection{Further discussion regarding model $P_{1}$}

This section discusses the problem of uniqueness of solution to model $P_{1}$

In Section 4.1, we obtain the optimal solution(s) to model $P_{1}$. However, in some situations, the optimal solution(s) to $P_{1}$ is not unique. Particular, some of the optimal solutions are not reasonable enough (in the 
sense of consistency). Here, we use an example, i.e., Example 1, to demonstrate this issue.

Example 1. In this example, three DMs $E=\left\{e_{1}, e_{2}, e_{3}\right\}$, four alternatives $X=\left\{x_{1}, x_{2}, x_{3}, x_{4}\right\}$, and three attributes $A=\left\{a_{1}, a_{2}, a_{3}\right\}$ are involved. The normalized multiple attribute decision matrix, $\bar{V}$, is given below:

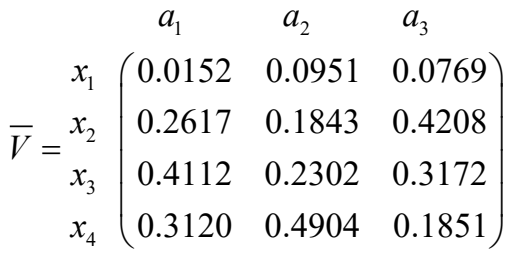

Miller [30] has demonstrated that an individual cannot simultaneously compare more than $7 \pm 2$ objects without confusion. The used linguistic term set with $\mathrm{g}+1$ symmetry linguistic terms is a $g / 2+1$ gradations. In this example the linguistic term set with 11 symmetry linguistic terms is used by DMs to express their preferences on alternatives.

$S=\left\{s_{0}=\right.$ extremely poorer, $s_{1}=$ much poorer, $s_{2}=$ poorer, $s_{3}=$ moderately poorer, $s_{4}=$ weakly poorer, $s_{5}=$ fair, $s_{6}=$ weakly better, $s_{7}=$ moderately better, $s_{8}=$ better, $s_{9}=$ much better, $s_{10}=$ extremely better\}

Meanwhile, the DM $e_{k}$ supplies the linguistic preference relation, $L^{k}$, based on linguistic term set $S$.

$$
L^{1}=\left(\begin{array}{llll}
s_{5} & s_{6} & s_{3} & s_{8} \\
s_{4} & s_{5} & s_{3} & s_{10} \\
s_{7} & s_{7} & s_{5} & s_{7} \\
s_{2} & s_{0} & s_{3} & s_{5}
\end{array}\right), \quad L^{2}=\left(\begin{array}{cccc}
s_{5} & s_{8} & s_{4} & s_{4} \\
s_{2} & s_{5} & s_{3} & s_{1} \\
s_{6} & s_{7} & s_{5} & s_{6} \\
s_{6} & s_{9} & s_{4} & s_{5}
\end{array}\right),
$$

$$
L^{3}=\left(\begin{array}{llll}
s_{5} & s_{1} & s_{4} & s_{5} \\
s_{9} & s_{5} & s_{8} & s_{3} \\
s_{6} & s_{2} & s_{5} & s_{1} \\
s_{5} & s_{7} & s_{9} & s_{5}
\end{array}\right)
$$

Here, we set $\gamma=0.05, \Delta=2, \lambda=\left(\frac{1}{3}, \frac{1}{3}, \frac{1}{3}\right)^{T}$ and $\alpha=0.5$. Using $L^{1}, L^{2}, L^{3}$ and $\bar{V}$ as the inputs of model $P_{1}$, we can obtain a set of optimal solutions. Here, we list two of them: $\Omega_{1}$ and $\Omega_{2}$, which are provided in Table 1 .

Based on $\Omega_{1}$ and $\Omega_{2}$, we can transform $L^{1}, L^{2}$ and $L^{3}$ into $P^{1}, P^{2}$ and $P^{3}$, respectively, and they are listed below:

(1) $P^{1}, P^{2}$ and $P^{3}$ under $\Omega_{1}$

$$
\begin{gathered}
P^{1}=\left(\begin{array}{cccc}
0.5 & 0.55 & 0.4 & 0.65 \\
0.45 & 0.5 & 0.4 & 0.9217 \\
0.6 & 0.6 & 0.5 & 0.6 \\
0.35 & 0.0783 & 0.4 & 0.5
\end{array}\right) \\
P^{2}=\left(\begin{array}{cccc}
0.5 & 0.6508 & 0.4492 & 0.4492 \\
0.3492 & 0.5 & 0.3992 & 0.1889 \\
0.5508 & 0.6008 & 0.5 & 0.5508 \\
0.5508 & 0.8111 & 0.4492 & 0.5
\end{array}\right) \\
P^{3}=\left(\begin{array}{cccc}
0.5 & 0.0939 & 0.3 & 0.5 \\
0.9061 & 0.5 & 0.8 & 0.25 \\
0.7 & 0.2 & 0.5 & 0.0939 \\
0.5 & 0.75 & 0.9061 & 0.5
\end{array}\right)
\end{gathered}
$$

Table 1 The individual numerical scales in $\Omega_{1}$ and $\Omega_{2}$

\begin{tabular}{ccccccccccccc}
\hline & & $s_{0}$ & $S_{1}$ & $s_{2}$ & $s_{3}$ & $s_{4}$ & $s_{5}$ & $s_{6}$ & $s_{7}$ & $s_{8}$ & $s_{9}$ & $s_{10}$ \\
\hline \multirow{3}{*}{$\Omega_{1}$} & $N S^{1}\left(s_{i}\right)$ & 0.0783 & 0.1893 & 0.35 & 0.4 & 0.45 & 0.5 & 0.55 & 0.6 & 0.65 & 0.8107 & 0.9217 \\
& $N S^{2}\left(s_{i}\right)$ & 0.0754 & 0.1889 & 0.3492 & 0.3992 & 0.4492 & 0.5 & 0.5508 & 0.6008 & 0.6508 & 0.8111 & 0.9246 \\
& $N S^{3}\left(s_{i}\right)$ & 0.0297 & 0.0939 & 0.2 & 0.25 & 0.3 & 0.5 & 0.7 & 0.75 & 0.8 & 0.9061 & 0.9703 \\
\hline \multirow{2}{*}{$\Omega_{2}$} & $N S^{1}\left(s_{i}\right)$ & 0.2 & 0.2628 & 0.35 & 0.4 & 0.45 & 0.5 & 0.55 & 0.6 & 0.65 & 0.7372 & 0.8 \\
& $N S^{2}\left(s_{i}\right)$ & 0.0788 & 0.2510 & 0.3492 & 0.3992 & 0.4492 & 0.5 & 0.5508 & 0.6008 & 0.6508 & 0.7490 & 0.9212 \\
& $N S^{3}\left(s_{i}\right)$ & 0.0310 & 0.0939 & 0.2 & 0.25 & 0.3 & 0.5 & 0,7 & 0.75 & 0.8 & 0.9061 & 0.9690 \\
\hline
\end{tabular}


(2) $P^{1}, P^{2}$ and $P^{3}$ under $\Omega_{2}$

$$
\begin{gathered}
P^{1}=\left(\begin{array}{cccc}
0.5 & 0.55 & 0.4 & 0.65 \\
0.45 & 0.5 & 0.4 & 0.8 \\
0.6 & 0.6 & 0.5 & 0.6 \\
0.35 & 0.2 & 0.4 & 0.5
\end{array}\right) \\
P^{2}=\left(\begin{array}{cccc}
0.5 & 0.6508 & 0.4492 & 0.4492 \\
0.3492 & 0.5 & 0.3992 & 0.251 \\
0.5508 & 0.6008 & 0.5 & 0.6008 \\
0.5508 & 0.749 & 0.4492 & 0.5
\end{array}\right) \\
P^{3}=\left(\begin{array}{cccc}
0.5 & 0.09309 & 0.3 & 0.5 \\
0.9061 & 0.5 & 0.8 & 0.25 \\
0.7 & 0.2 & 0.5 & 0.0939 \\
0.5 & 0.75 & 0.9061 & 0.5
\end{array}\right)
\end{gathered}
$$

According to definition 7 , we can obtain the consistency levels of the transformed additive preference relations $P^{k}(k=1,2,3)$ under $\Omega_{1}$ and $\Omega_{2}$, respectively, which are listed in Table 2 .

Table 2. The consistency levels of $P^{k}(\mathrm{k}=1,2,3)$ under $\Omega_{1}$ and $\Omega_{2}$, respectively

\begin{tabular}{r|ccc}
\hline & $P^{1}$ & $P^{2}$ & $P^{3}$ \\
\hline$C C I\left(P^{k}\right)$ under & 0.1572 & $\mathbf{0 . 2 7 5 5}$ & 0.2500 \\
$\Omega_{1}$ & 0.1167 & 0.2278 & $\mathbf{0 . 2 5 0 0}$ \\
\hline$C C I\left(P^{k}\right)$ under \\
$\Omega_{2}$
\end{tabular}

Example 1 shows that: (1) In some situations, optimal solutions to $P_{1}$ are not unique; and (2) $\max _{\Omega_{1}, k}\left\{C C I\left(P^{k}\right)\right\} \geq \max _{\Omega_{2}, k}\left\{C C I\left(P^{k}\right)\right\} \quad, \quad$ this implies that the optimal solution $\Omega_{2}$ is better than $\Omega_{1}$ in the sense of consistency.

Therefore, it is necessary to further optimize the optimal solutions to $P_{1}$. Let $M^{*}$ be the optimal objective function of model $P_{1}$, and let $\Omega=\left\{\Omega_{1}, \Omega_{2}, \ldots, \Omega_{T}\right\}$ be a set of optimal solution(s) to model $P_{1}$. Then, using the optimization model,

$$
\min _{\Omega_{s} \in \Omega} \max _{k} C C I\left(P^{k}\right)
$$

yields the unique optimal solution. Model (18) further minimizes the maximal consistency index of the transformed additive preference relations.

Further, model (18) can be rewritten as follows:

$$
\begin{aligned}
& \min \max _{k} C C I\left(P^{k}\right)
\end{aligned}
$$

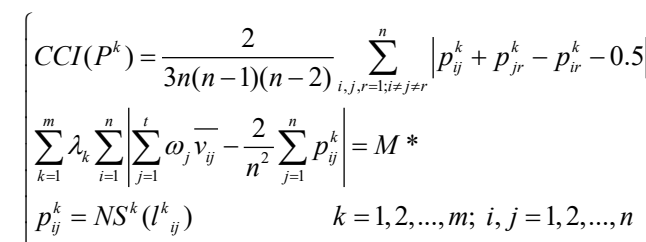

$$
\begin{aligned}
& N S^{k}\left(s_{i}\right) \in[0,(i+\Delta) / g] \quad k=1,2, \ldots, m ; i=0,1, \ldots, \Delta-1 \\
& \text { s.t. }\left\{\frac{(i-\Delta)}{g} \leq N S^{k}\left(s_{i}\right) \leq \frac{(i+\Delta)}{g} \quad k=1,2, \ldots, m ; i=\Delta, \Delta+1, \ldots, \frac{g}{2}\right. \\
& N S^{k}\left(s_{i+1}\right)-N S^{k}\left(s_{i}\right) \geq \gamma \quad k=1,2, \ldots, m ; i=0,1, \ldots, \frac{g}{2} \\
& N S^{k}\left(s_{i}\right)+N S^{k}\left(s_{g-i}\right)=1 \quad k=1,2, \ldots, m ; i=0,1,2, \ldots, \frac{g}{2} \\
& \sum_{j=1}^{t} \omega_{j}=1 \\
& \omega_{j} \geq 0 \quad j=1,2, \ldots, t
\end{aligned}
$$

In model (19), $N S^{k}\left(s_{i}\right)$ and $\omega=\left(\omega_{1}, \omega_{2}, \ldots, \omega_{t}\right)^{T}$ are decision variables. To solve model (19), Theorem 2 is presented.

Theorem 2. By introducing a variable $\theta \geq 0$, then model (19) can be equally transformed into the following model:

$$
\begin{aligned}
& \min \theta \\
& \left\{\begin{array}{l}
\frac{2}{3 n(n-1)(n-2)} \sum_{i=1}^{n} \sum_{j=1}^{n} \sum_{r=1}^{n}\left|p_{i j}^{k}+p_{j r}^{k}-p_{i r}^{k}-0.5\right| \leq \theta \quad k=1,2, \ldots, m \\
\sum_{k=1}^{m} \lambda_{k} \sum_{i=1}^{n}\left|\sum_{j=1}^{t} \omega_{j} \bar{v}_{i j}-\frac{2}{n^{2}} \sum_{j=1}^{n} p_{i j}^{k}\right|=M^{*}
\end{array}\right. \\
& \begin{cases}p_{i j}^{k}=N S^{k}\left(l_{i j}\right) & k=1,2, \ldots, m ; i, j=1,2, \ldots, n \\
N S^{k}\left(s_{i}\right) \in[0,(i+\Delta) / g] & k=1,2, \ldots, m ; i=0,1, \ldots, \Delta-1 \\
(i-\Delta) & \end{cases} \\
& \text { s.t. }\left\{\frac{(i-\Delta)}{g} \leq N S^{k}\left(s_{i}\right) \leq \frac{(i+\Delta)}{g} \quad k=1,2, \ldots, m ; i=\Delta, \Delta+1, \ldots, \frac{g}{2}\right. \\
& N S^{k}\left(s_{i+1}\right)-N S^{k}\left(s_{i}\right) \geq \gamma \quad k=1,2, \ldots, m ; i=0,1, \ldots, \frac{g}{2} \\
& N S\left(s_{i}\right)+N S\left(s_{g-i}\right)=1 \quad i=0,1,2, \ldots, \frac{g}{2} \\
& \sum_{j=1}^{t} \omega_{j}=1 \\
& \omega_{j} \geq 0 \quad j=1,2, \ldots, t
\end{aligned}
$$

Proof. In model (20), constraint (1) guarantees that $\theta \geq \max _{k}\left\{C C I\left(P^{k}\right)\right\}$. According to the objective function of model (20), any feasible solutions with $\theta>\max _{k}\left\{C C I\left(P^{k}\right)\right\}$ are not the optimal solution to model (20). Thus, $\theta=\max _{k}\left\{C C I\left(P^{k}\right)\right\}$. Consequently, 
model (19) can be equally transformed into the model (20). This completes the proof of Theorem 2.

Similar to the transformation process of model $P_{1}$, model (20) can be transformed into a linear programming model that can be easily solved. For space limitations, we omit the transformation process of model (20).

\subsection{Numerical example}

In this section, we use a numerical example to verify the effectiveness of the proposed approach.

In this example, there are three DMs $E=\left\{e_{1}, e_{2}, e_{3}\right\}$, four alternatives $X=\left\{x_{1}, x_{2}, x_{3}, x_{4}\right\}$, and four attributes $A=\left\{a_{1}, a_{2}, a_{3}, a_{4}\right\}$. In particular, $a_{1}, a_{3}, a_{4}$ are benefit attributes, and $a_{2}$ is a cost attribute. The multiple attribute matrix, $V$, is provided in Table 3.

Meanwhile, DMs $E=\left\{e_{1}, e_{2}, e_{3}\right\}$ provide their linguistic preference relations $\left\{L^{1}, L^{2}, L^{3}\right\}$ on alternatives $X=\left\{x_{1}, x_{2}, x_{3}, x_{4}\right\}$ using the following linguistic term set:

$S=\left\{s_{0}=\right.$ extremely poorer, $s_{1}=$ much poorer, $s_{2}=$ poorer, $s_{3}=$ moderately poorer, $s_{4}=$ weakly poorer, $s_{5}=$ fair, $s_{6}=$ weakly better, $s_{7}=$ moderately better, $s_{8}=$ better, $s_{9}=$ much better, $s_{10}=$ extremely better\}

Table 3 The multiple attribute decision matrix $V$

\begin{tabular}{ccccc}
\hline & $a_{1}$ & $a_{2}$ & $a_{3}$ & $a_{4}$ \\
\hline$x_{1}$ & 16 & 1 & 4 & 3 \\
$x_{2}$ & 32 & 1 & 1 & 6 \\
$x_{3}$ & 8 & 1 & 2 & 3 \\
$x_{4}$ & 24 & 3 & 3 & 18 \\
\hline
\end{tabular}

The linguistic preference relations $\left\{L^{1}, L^{2}, L^{3}\right\}$ are listed below:

$$
\begin{gathered}
L^{1}=\left(\begin{array}{llll}
s_{5} & s_{3} & s_{8} & s_{9} \\
s_{7} & s_{5} & s_{9} & s_{7} \\
s_{2} & s_{1} & s_{5} & s_{6} \\
s_{1} & s_{3} & s_{4} & s_{5}
\end{array}\right), L^{2}=\left(\begin{array}{cccc}
s_{5} & s_{10} & s_{6} & s_{5} \\
s_{0} & s_{5} & s_{9} & s_{2} \\
s_{4} & s_{1} & s_{5} & s_{3} \\
s_{5} & s_{8} & s_{7} & s_{5}
\end{array}\right) \\
L^{3}=\left(\begin{array}{llll}
s_{5} & s_{6} & s_{6} & s_{6} \\
s_{4} & s_{5} & s_{7} & s_{2} \\
s_{4} & s_{3} & s_{5} & s_{2} \\
s_{4} & s_{8} & s_{8} & s_{5}
\end{array}\right)
\end{gathered}
$$

We set $\gamma=0.05, \Delta=2, \quad \lambda=\left(\frac{1}{3}, \frac{1}{3}, \frac{1}{3}\right)^{T}$ and $\alpha=0.5$ in this example. In the following, we use the proposed method to help DMs to yield the ranking of the alternatives.

According to Eqs. (6) and (7), $V$ is normalized into $\bar{V}=\left(\overline{v_{i j}}\right)_{n \times t}$ :

$$
\begin{aligned}
& \begin{array}{llll}
a_{1} & a_{2} & a_{3} & a_{4}
\end{array} \\
& \bar{V}=\begin{array}{c}
x_{1} \\
x_{2} \\
x_{3} \\
x_{4}
\end{array}\left(\begin{array}{llll}
0.2 & 0.3 & 0.4 & 0.1 \\
0.4 & 0.3 & 0.1 & 0.2 \\
0.1 & 0.3 & 0.2 & 0.1 \\
0.3 & 0.1 & 0.3 & 0.6
\end{array}\right)
\end{aligned}
$$

The model $P_{1}$ is used to get $M^{*}=0.1792$. Further, the individual numerical scales with PIS and attribute weights can be obtained by solving model (19), which are provided below:

$$
\omega=(0.172,0.330,0.341,0.157)^{T}
$$

Based on Table 4, the linguistic preference relations $L^{1}, L^{2}$ and $L^{3}$ are transformed into additive preference relations $P^{1}, P^{2}$ and $P^{3}$, respectively.

$$
\begin{aligned}
P^{1} & =\left(\begin{array}{cccc}
0.5 & 0.4 & 0.667 & 0.717 \\
0.6 & 0.5 & 0.717 & 0.6 \\
0.333 & 0.283 & 0.5 & 0.55 \\
0.283 & 0.4 & 0.45 & 0.5
\end{array}\right), \\
P^{2} & =\left(\begin{array}{cccc}
0.5 & 0.8 & 0.55 & 0.5 \\
0.2 & 0.5 & 0.75 & 0.35 \\
0.45 & 0.25 & 0.55 & 0.4 \\
0.5 & 0.65 & 0.6 & 0.5
\end{array}\right), \\
P^{3} & =\left(\begin{array}{cccc}
0.5 & 0.583 & 0.583 & 0.583 \\
0.417 & 0.5 & 0.633 & 0.317 \\
0.417 & 0.367 & 0.5 & 0.317 \\
0.417 & 0.683 & 0.683 & 0.5
\end{array}\right)
\end{aligned}
$$

Use Eq. (8) to yield the objective preference vector, $O P V$ :

$$
O P V=(0.2855,0.2333,0.2000,0.2812)^{T}
$$

According to Eq. (9), we can obtain subjective preference vectors $S P V^{(k)} \quad(k=1,2,3)$ from additive preference relations $P^{k} \quad(k=1,2,3)$, and they are listed below:

$$
\begin{aligned}
& e_{1}: S P V^{(1)}=(0.2855,0.3021,0.2083,0.2041)^{T} \\
& e_{2}: S P V^{(2)}=(0.3059,0.2066,0.2041,0.2834)^{T}
\end{aligned}
$$




$$
e_{3}: S P V^{(3)}=(0.2687,0.2354,0.2104,0.2855)^{T}
$$

Following this, according to Eq. (4), the collective subjective preference vector $S P V$, can be obtained by aggregating $S P V^{(1)}, S P V^{(2)}$ and $S P V^{(3)}$ :

$$
S P V=(0.2867,0.2480,0.2076,0.2577)^{T}
$$

Further, the collective preference vector $P V$, can be obtained by fusing $O P V$ and $S P V$ using Eq. (5):

$$
P V=(0.2861,0.2407,0.2037,0.2695)^{T}
$$

Based on $P V$, the ranking of the four alternatives can obtained, that is $x_{1}>x_{4}>x_{2}>x_{3}$

Table 4 The individual numerical scales with PIS

\begin{tabular}{cccccccccccc}
\hline & $s_{0}$ & $S_{1}$ & $S_{2}$ & $S_{3}$ & $S_{4}$ & $S_{5}$ & $s_{6}$ & $S_{7}$ & $S_{8}$ & $s_{9}$ & $S_{10}$ \\
\hline$N S^{1}\left(s_{i}\right)$ & 0.1 & 0.283 & 0.333 & 0.4 & 0.45 & 0.5 & 0.55 & 0.6 & 0.667 & 0.717 & 0.9 \\
$N S^{2}\left(s_{i}\right)$ & 0.2 & 0.25 & 0.35 & 0.4 & 0.45 & 0.5 & 0.55 & 0.6 & 0.65 & 0.75 & 0.8 \\
$N S^{3}\left(s_{i}\right)$ & 0.075 & 0.185 & 0.317 & 0.367 & 0.417 & 0.5 & 0.583 & 0.633 & 0.683 & 0.815 & 0.925 \\
\hline
\end{tabular}

\section{Numerical and simulation analysis}

In this section, we present several comparison criteria, and propose numerical and simulation analysis to discuss the validity of our proposal.

\subsection{Comparison criteria}

In the MAGDM with preference information on alternatives, there are two kinds of preference information, namely: objective and subjective preference information. Naturally, we hope that the objective and subjective preference information are as consistent as possible. Following this idea, we present the following criteria to evaluate the validity of our proposal: the deviation between the objective and subjective preference vectors, and the deviation between the objective and subjective preference rankings of alternatives $[16,45]$. In the extant literature regarding the GDM, Manhattan distance and Euclidean distance have been widely used to measure the distance between preference vectors. Thus, Manhattan distance and Euclidean distance are adopted to measure the deviation between the objective and subjective preference vectors in this study. In addition, the consistency is a vital basis for GDM with preference relations. Thus, we consider the consistency of the transformed additive preference relations as a criterion to evaluate the validity of our proposal. The basic knowledge regarding the consistency issue of additive preference relations have been introduced in Definition 7 (section 2.3). The proposed comparison criteria except for the consistency of additive preference relations are introduced below.

(1) The Manhattan distance between the objective and subjective preference vectors

The Manhattan distance, $M D$, between the objective preference vector, $O P V$, and the subjective preference vector, $S P V$, is computed as follows:

$$
M D=\sum_{i=1}^{n}\left|O P V_{i}-S P V_{i}\right|
$$

where $M D \in[0,2]$. The smaller $M D$ value denotes the better consistency between objective and subjective preference information, and the more effective of the approach.

(2) The Euclidean distance between the objective and subjective preference vectors

The Euclidean distance, $E D$, between the objective preference vector, $O P V$, and the subjective preference vector, $S P V$, is computed as follows:

$$
E D=\sqrt{\sum_{i=1}^{n}\left(O P V_{i}-S P V_{i}\right)^{2}}
$$

where $E D \in[0,2]$. The smaller $E D$ value signifies the better consistency between objective and subjective preference information, and the more effective of the approach.

(3) The deviation between objective and subjective preference rankings of alternatives

Let $O=\left(o_{1}, o_{2}, \ldots, o_{n}\right)^{T}$ be the objective preference ordering derived from the objective 
preference vector $O P V$, where, if $O P V_{i}$ $(i=1,2, \ldots, n)$ is $j$ largest in $O P V$, then $o_{i}=j$ $(i=1,2, \ldots, n)$. Using a similar way, the objective preference ordering $\bar{O}=\left(\overline{o_{1}}, \overline{o_{2}}, \ldots, \overline{o_{n}}\right)^{T} \quad$ can be obtained from the subjective preference vector $S P V$.

Let $D$ [24] be the deviation between objective and subjective preference rankings of alternatives, which can be calculated by:

$$
D=2 \sum_{i=1}^{n} \frac{\left|o_{i}-\overline{o_{i}}\right|}{n^{2}}
$$

where $D \in[0,1]$. The smaller $D$ value indicates the better consistency between the objective and subjective preference information.

\subsection{Numerical analysis}

The existing approach for MAGDM with linguistic preference relations on alternatives is based on the fixed numerical scale. In our model $P_{1}$, if the additive preference relation $P^{k}=\left(p_{i j}^{k}\right)_{n \times n}$ associated with linguistic preference relation $L^{k}=\left(l_{i j}^{k}\right)_{n \times n}$ is previously assumed known, then model $P_{1}$ can be reduced to the existing approach. In the following, we use an example to show the difference between our proposal with the existing approach.

The data used in this example is derived from Section 4.3. Using the fixed numerical scale, i.e., $N S^{(k)}\left(s_{i}\right)=\frac{i}{g}$, we can transform linguistic preference relations $L^{k}=\left(l_{i j}^{k}\right)_{n \times n}$ into the following additive preference relations:

$$
\begin{gathered}
\overline{P^{1}}=\left(\begin{array}{cccc}
0.5 & 0.3 & 0.8 & 0.9 \\
0.7 & 0.5 & 0.9 & 0.7 \\
0.2 & 0.1 & 0.5 & 0.6 \\
0.1 & 0.3 & 0.4 & 0.5
\end{array}\right), \quad \overline{P^{2}}=\left(\begin{array}{cccc}
0.5 & 1 & 0.6 & 0.5 \\
0 & 0.5 & 0.9 & 0.2 \\
0.4 & 0.1 & 0.5 & 0.3 \\
0.5 & 0.8 & 0.7 & 0.5
\end{array}\right), \\
\overline{P^{3}} \\
=\left(\begin{array}{llll}
0.5 & 0.6 & 0.6 & 0.6 \\
0.4 & 0.5 & 0.7 & 0.2 \\
0.4 & 0.3 & 0.5 & 0.2 \\
0.4 & 0.8 & 0.8 & 0.5
\end{array}\right)
\end{gathered}
$$

Taking $\overline{P^{1}}, \overline{P^{2}}$ and $\overline{P^{3}}$ as the inputs of the model $P_{1}$, we can obtain the attributes weight vector, that is,

$$
\omega=(0.249,0.344,0.162,0.245)^{T}
$$

Based on the obtained attribute weights, the objective preference vector is obtained from Eq. (8),

$$
O P V=(0.2423,0.2681,0.1850,0.3046)^{T}
$$

According to Eq. (9), we can obtain subjective preference vectors $S P V^{(k)} \quad(k=1,2,3)$ from additive preference relations $\overline{P^{k}} \quad(k=1,2,3)$, and they are listed below:

$$
\begin{aligned}
& e_{1}: S P V^{(1)}=\left(\begin{array}{ll}
0.3125,0.3500,0.1750,0.1625
\end{array}\right)^{T} \\
& e_{2}: S P V^{(2)}=(0.3250,0.2000,0.1625,0.3125)^{T} \\
& e_{3}: S P V^{(3)}=\left(\begin{array}{ll}
0.2875,0.2250,0.1750,0.3125
\end{array}\right)^{T}
\end{aligned}
$$

According to Eq. (4), the collective subjective preference vector $S P V$, can be obtained by aggregating $S P V^{(1)}, S P V^{(2)}$ and $S P V^{(3)}$ :

$$
S P V=(0.3083,0.2583,0.1708,0.2625)^{T}
$$

Further, the collective preference vector $P V$, can be obtained by fusing $O P V$ and $S P V$ using Eq.(5):

$$
P V=(0.2753,0.2632,0.1779,0.2836)^{T}
$$

Based on $P V$, the ranking of alternatives can be obtained, that is $x_{4}>x_{1}>x_{2}>x_{3}$.

From this example and the numerical example presented in Section 4.3, we find that the collective solutions, i.e., the collective preference rankings of alternatives, under our proposal and traditional approach are different. This finding means that the PIS will influence the decision result. In the following, we compare these two approaches based on the four comparison criteria presented in Section 5.1. The comparison results are listed in Table 5.

Table 5 The comparison results between MAGDM approaches with PIS and FNS

\begin{tabular}{c|cccc}
\hline & MD & ED & D & CCI \\
\hline $\begin{array}{c}\text { MAGDM approach } \\
\text { with PIS }\end{array}$ & 0.0472 & 0.0289 & 0 & 0.1148 \\
\hline $\begin{array}{c}\text { MAGDM approach } \\
\text { with FNS }\end{array}$ & 0.1321 & 0.0802 & 0.5 & 0.1889 \\
\hline
\end{tabular}

From table 5, we find that our PIS based approach has a better decision efficiency under all criteria compared with the MAGDM approach with the fixed numerical scale. 


\subsection{Simulation analysis}

(1) The comparison method

In this section, the existing approach for MAGDM with linguistic preference relations on alternatives is introduced. Then, a simulation method is designed to compare our proposal with the existing approach.

The basic idea of the simulation method is as follows:

We randomly generate the objective preference information $V=\left(v_{i j}\right)_{n \times t}$ and subjective preference information $L^{k}=\left(l_{i j}^{k}\right)_{n \times n}$. Then, we take the generated $V=\left(v_{i j}\right)_{n \times t}$ and $P^{k}=\left(p_{i j}^{k}\right)_{n \times n}$ as the inputs of our proposal (i.e., the MAGDM approach with the PIS) and the traditional method (i.e., the MAGDM approach with the FNS) to obtain the objective and subjective preference vectors, objective and subjective rankings and the transformed additive preference relations, respectively. Further, we can obtain the $M D, E D$, $D$ and $C C I$ values under these two approaches, respectively. The specific steps of the simulation method are provided in Table 6 .

Table 6 Simulation method

Input: $n, m, t, \alpha \lambda=\left(\lambda_{1}, \lambda_{2}, \ldots, \lambda_{m}\right)^{T}$ and $S=\left\{s_{g} \mid g=0,1, \ldots, g\right\}$

Output: $D^{(P I S)}, D^{(F N S)}, E D^{(P I S)}, E D^{(F N S)}$, $M D^{(P I S)}, M D^{(F N S)}, C C I^{(P I S)}$ and $C C I^{(F N S)}$.

Step 1: We generate multiple attribute decision matrix $V=\left(v_{i j}\right)_{n \times t}$ and linguistic preference relations $L^{k}=\left(l_{i j}^{k}\right)_{n \times n}$ with weak stochastic transitivity, where $v_{i j}$ is randomly and uniformly generated from $[0,1]$, and $l_{i j}^{k}$ is randomly and uniformly generated from $S=\left\{s_{g} \mid g=0,1, \ldots, g\right\}$.

Step 2: Use Eqs. (6) and (7) to transform $V=\left(v_{i j}\right)_{n \times t}$ into the normalized multiple attribute decision matrix $\bar{V}=\left(\overline{v_{i j}}\right)_{n \times t}$.

Step 3: Take $\bar{V}=\left(\overline{v_{i j}}\right)_{n \times t}$ and $L^{k}=\left(l_{i j}^{k}\right)_{n \times n}$ as the inputs of models (16) and (19) to determine the numerical scale $N S^{k}\left(s_{i}\right)$ of linguistic term $s_{i}$ and attribute weights vector $\omega$. Based on $N S^{k}\left(s_{i}\right)$, the linguistic preference relation $L^{k}=\left(l_{i j}^{k}\right)_{n \times n}$ can be transformed into additive preference relation
$P^{k}=\left(p_{i j}^{k}\right)_{n \times n}$. According to $\bar{V}=\left(\overline{v_{i j}}\right)_{n \times t}$ and the obtained attribute weights vector $\omega$, we can get the objective preference vector using Eq. (8). Meanwhile, employing Eqs. (9) and (4) result in the collective subjective preference vector from $P^{k}=\left(p_{i j}^{k}\right)_{n \times n}$. Finally, utilizing Eqs. (3), (21)-(23) yields $M D, E D$, $D$ and $C C I$, which are denoted as $D^{(P I S)}, E D^{(P I S)}$, $M D^{(P I S)}$, and $C C I^{(P I S)}$, respectively.

Step 4: Transform the linguistic preference information $L^{k}=\left(l_{i j}^{k}\right)_{n \times n}$ into numerical preference information $\overline{P^{k}}=\left(\overline{p_{i j}^{k}}\right)_{n \times n}$ according to the fixed numerical scale, where if $l_{i j}^{k}=s_{t}$, then $\overline{p_{i j}^{k}}=N S^{k}\left(s_{t}\right)=t / g$. Then, take $\bar{V}=\left(\overline{v_{i j}}\right)_{n \times t}$ and $\overline{P^{k}}=\left(\overline{p_{i j}^{k}}\right)_{n \times n}$ as the inputs of model (16), we can obtain attribute weights vector $\omega$. Following this, based on $\bar{V}=\left(\overline{v_{i j}}\right)_{n \times t}$ and the obtained attribute weights vector $\omega$, we can get the objective preference vector according to Eq. (8). Next, adopting Eqs. (9) and (4) yields the collective subjective preference vector from $\overline{P^{k}}=\left(\overline{p_{i j}^{k}}\right)_{n \times n}$. Finally, according to Eqs. (3), (21)-(23), $M D, E D, D$ and $C C I$, can be obtained, which are denoted as $D^{(F N S)}, E D^{(F N S)}, M D^{(F N S)}$, and $C C I^{(F N S)}$, respectively.

Step 5: Output $D^{(P I S)}, D^{(F N S)}, E D^{(P I S)}$, $E D^{(F N S)}, M D^{(P I S)}, M D^{(F N S)}, C C I^{(P I S)}$ and $C C I^{(F N S)}$.

(2) Comparison results

Let $S=\left\{s_{i} \mid i=0,1,2, \ldots, 10\right\}, \quad \lambda=\left(\frac{1}{3}, \frac{1}{3}, \frac{1}{3}\right)^{T}, \quad t=4$ and $\alpha=0.5$. When setting different parameters $m$ and $n$, we run this simulation method 1000 times to obtain the average $M D, E D, D$ and $C C I$ values under MAGDM approach with PIS and MAGDM approach with FNS, respectively, and they are respectively described in Figs. 2-5.

From Figs. 2-5, we have the following observations. The Manhattan distance (MD) and Euclidean distance (ED) between the objective and subjective preference vectors, the deviation between objective and subjective preference rankings of alternatives (D), and the consistency of the transformed additive preference relations (CCI) in our proposed approach are obviously lower than those in the traditional approach with PNS, respectively. This 
finding implies that the proposed approach can improve the decision efficiency by taking PIS into account.

Note 2: In our proposal, the linguistic preference relations are transformed into additive preference relations, which are based on fuzzy numerical scales with PIS. So, we compare our proposal with the traditional method with fixed fuzzy numerical scales $[12,16,17]$. In the future, we plan to investigate the use of interval fuzzy numerical scales in the PIS based MAGDM framework, and compare it with the linguistic decision model with the fixed interval fuzzy numerical scales.

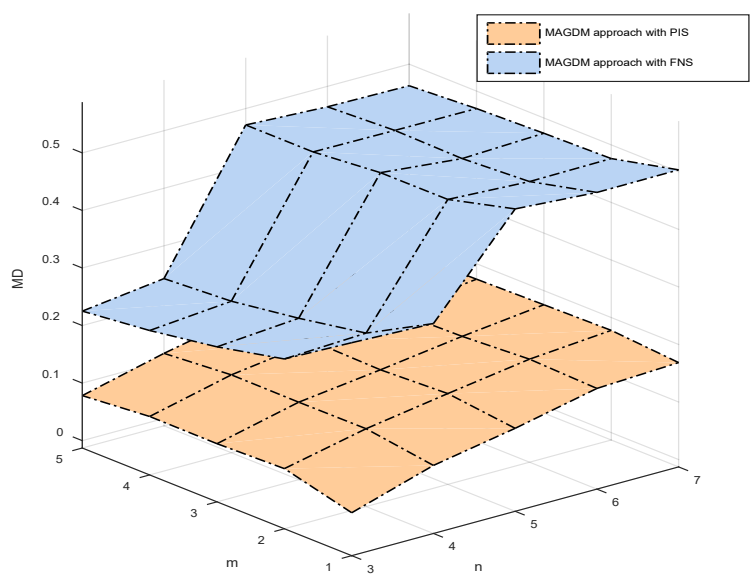

Fig.2. Average $M D$ value

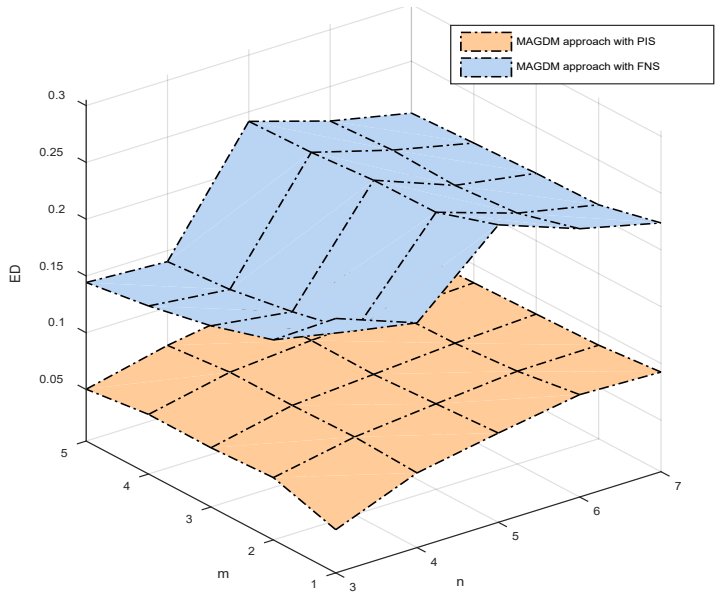

Fig.3. Average $E D$ value

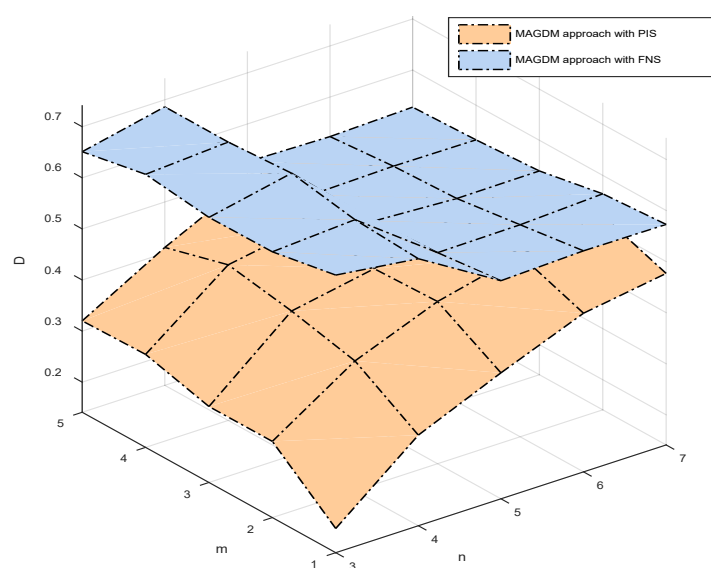

Fig. 4. Average $D$ value

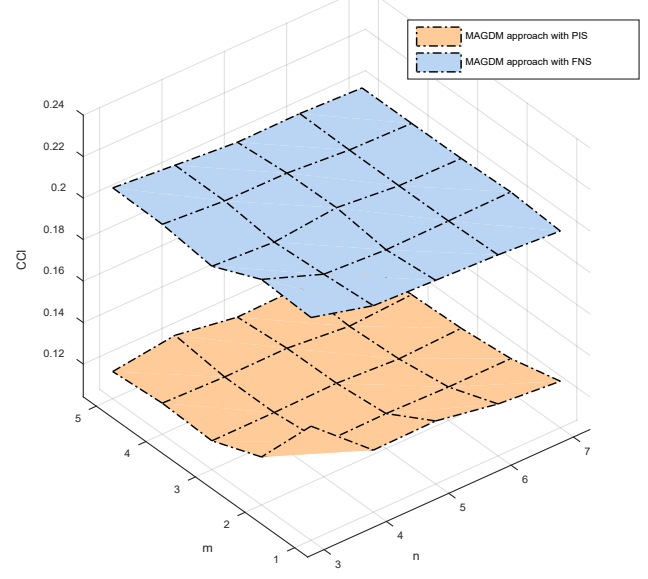

Fig. 5. Average $C C I$ value

\section{Conclusion}

In this study, we propose a PIS based decision framework for MAGDM with linguistic preference relations on alternatives. The main points presented are as follows.

(1) The extant researches regarding the MAGDM with preference information on alternatives assumed that the DMs provide their preference information using a numerical way. This study assumes that DMs provide their preference information over alternatives using a linguistic way rather than a numerical way, which is closer to the realistic decision scenario.

(2) In decision making with linguistic preference information, PIS are important elements that cannot be ignored due to their influences on the final decision. This study takes the PIS into account, and proposes a 
PIS based MAGDM framework to improve the decision efficiency.

(3) We build a two-stage-based optimization model to obtain the unique individual numerical scale with the PIS. Moreover, we propose an approach to transform the proposed optimization-based optimization models into linear programming models that can be easily solved.

(4) We design detailed numerical and simulation experiments to verify the validity of the PIS based MAGDM framework. The numerical and simulation experiments show that our proposal has a better decision efficiency compared with the existing MAGDM framework.

Meanwhile, we argue that there are two interesting research paths for the future:

(1) In our proposal, each element in the preference relation is mapped to an exact number. However, the preference relation is usually with uncertainty. To deal with this issue, the uncertain numerical scale (interval numerical scale) has been proposed and used in the linguistic decision making [5], further studies should discuss the uses of the interval numerical scale in the PIS based MAGDM framework.

(2) In addition, the consensus has become a hot topic in MAGDM [4, 8, 10, 13, 46, 50]. We argue that it will be interesting in any future research to investigate the consensus issues in the PIS based MAGDM framework.

\section{Acknowledgements}

This work was supported by the grant (No. 71571124) from NSF of China, and the grant (No. 2017B07514) from "the Fundamental Research Funds for the Central Universities".

\section{References}

1. S. Alonso, F.J. Cabrerizo, F. Chiclana, F. Herrera, E. Herrera-Viedma, Group decision-making with incomplete fuzzy linguistic preference relations, International Journal of Intelligent Systems 24 (2009) 201-222

2. X. Chen, H.J. Zhang, Y.C. Dong, The fusion process with heterogeneous preference structures in group decision making: A survey, Information Fusion 24 (2015) 72-83.

3. F. Chiclana, E. Herrera-Viedma, S. Alonso, F. Herrera, Cardinal consistency of reciprocal preference relations: a characterization of multiplicative transitivity, IEEE Transactions on Fuzzy Systems 17 (2009) 14-23.

4. Y.C. Dong, Z.G. Ding, L. Martínez, F. Herrera, Managing consensus based on leadership in opinion dynamics,
Information Sciences 397-398 (2017) 187-205.

5. Y.C. Dong, E. Herrera-Viedma, Consistency-driven automatic methodology to set interval numerical scales of 2-tuple linguistic term sets and its use in the linguistic GDM with preference relation, IEEE Transactions on Cybernetics 45 (4) (2015) 780-792.

6. Y.C. Dong, C.C. Li, F. Herrera, Connecting the linguistic hierarchy and the numerical scale for the 2-tuple linguistic model and its uses to deal with hesitant unbalanced linguistic information, Information Sciences 367-368 (2016) 259-278.

7. Y.C. Dong, Y.T. Liu, H.M. Liang, F. Chiclana, E. Herrera-Viedma, Strategic weight manipulation in multiple attribute decision making, Omega 75 (2018) 154-164.

8. Y.C. Dong, C.C. Li, Y. Xu, X. Gu, Consensus-based group decision making under multi-granular unbalanced 2-tuple linguistic preference relations, Group Decision and Negotiation 24 (2) (2015) 217-242.

9. Y.C. Dong, G.Q. Zhang, W.C. Hong, S. Yu, Linguistic computational model based on 2-tuples and intervals, IEEE Transactions on Fuzzy Systems 21 (2013) 1006-1018.

10. Y.C. Dong, H.J. Zhang, E. Herrera-Viedma, Consensus reaching model in the complex and dynamic MAGDM problem, Knowledge-Based Systems $106 \quad$ (2016) 206-219.

11. J.S. Dyer, P.C. Fishburn, R.E. Steuer, J. Wallenius, S. Zionts, Multiple criteria decision making, multiattribute utility theory: the next ten years, Management Science 38(5) (1992) 645-654.

12. Z.P. Fan, D.F. Hu, S.H. Xiao, A method for multiple attribute decision-making with the fuzzy preference relation on alternatives, Computer \& Industrial Engineering 46 (2004) 321-327.

13. Z.W. Gong, C. Xu, F. Chiclana, X.X. Xu, Consensus measure with multi-stage fluctuation utility based on china's urban demolition negotiation, Group Decision and Negotiation 26 (2016) 1-29.

14. E. Herrera-Viedma, F. Herrera, F. Chiclana, A consensus model for multiperson decision making with different preference structures, IEEE Transactions on Systems, Man and Cybernetics Part A: Systems and Humans 32 (2002) 394-402.

15. E. Herrera-Viedma, F. Herrera, F. Chiclana, M. Luque, Some issues on consistency of fuzzy preference relations, European Journal of Operational Research 154 (1) (2004) 98-109.

16. E. Herrera-Viedma, F. Chiclana, F. Herrera, S. Alonso, Group decision-making model with incomplete fuzzy preference relations based on additive consistency, IEEE Transactions on Systems, Man, and Cybernetics - part B: Cybernetics 37 (1) (2007) 176-189.

17. F. Herrera, L. Martinez, A 2-tuple fuzzy linguistic representation model for computing with words, IEEE Trans. Fuzzy Systems 8 (6) (2000) 746-752.

18. D.S. Hochbaum, A. Levin, Methodologies and algorithms 
for group-rankings decision, Management Science 52 (9) (2006) 1394-1408.

19. C.L. Hwang, K.P. Yoon, Multiple attribute decision making. Methods and applications. A state-of-the-art survey, Lecture Notes in Economics \& Mathematical Systems 375 (4) (1981) 1-531.

20. M. Kadziński, T. Tervonen, Robust multi-criteria ranking with additive value models and holistic pair-wise preference statements, European Journal of Operational Research 228 (2013) 169-180.

21. C.C. Li, Y.C Dong, F. Herrera, E. Herrera-Viedma, L. Martínez, Personalized individual semantics in computing with words for supporting linguistic group decision making. An application on consensus reaching, Information Fusion 33 (2017) 29-40.

22. C.C. Li, R.M. Rodriguez, L. Martinez, Y.C. Dong, F. Herrera, Consistency of hesitant fuzzy linguistic preference relations: An interval consistency index, Information Sciences 432 (2018) 347-361.

23. W.Q. Liu, Y.C. Dong, F. Chiclana, F.J. Cabrerizo, E. Herrera-Viedma, Group decision-making based on heterogeneous preference relations with self-confidence, Fuzzy Optimization and Decision Making 16 (2017) 429-447.

24. L. Martínez , F. Herrera, An overview on the 2-tuple linguistic model for computing with words in decision making: Extensions, applications and challenges, Information Sciences 207 (2012) 1-18.

25. L. Martínez, D. Ruan, F. Herrera, Computing with words in decision support systems: An overview on models and applications, International Journal of Computational Intelligence Systems 3 (4) (2010) 382-395.

26. J. Ma, Q. Zhang, Z.P. Fan, An approach to multiple attribute decision making based on preference information on alternatives. Fuzzy Sets and Systems 131 (2002) 101-106.

27. J.M. Mendel, D. Wu, Perceptual computing: Aiding people in making subjective Judgments, IEEE-Wiley press, John Wiley \& Sons, Inc., Hoboken, New Jersey (2010).

28. J.M. Mendel, L.A. Zadeh, E. Trillas, R.R. Yager, J. Lawry, H. Hagras, S. Guadarrama, What computing with words means to me: Discussion forum, IEEE Computational Intelligence Magazine 5 (1) (2010) 20-26.

29. J.M. Merigó, M. Casanovas, L. Martínez, Linguistic aggregation operators for linguistic decision making based on the dempster-shafer theory of evidence, International Journal of Uncertainty Fuzziness and Knowledge-Based Systems 18 (3) (2010) 287-304.

30. G.A. Miller, The magical number seven plus or minus two: Some limits on our capacity of processing information, Psychological Review 101(2) (1994) 343-352.

31. J.A. Morente-Molinera, I.J. Pérez, M.R. Ureña, E. Herrera-Viedma, On multi-granular fuzzy linguistic modelling in group decision making problems: a systematic review and future trends, Knowledge-Based
Systems 74 (2015) 49-60.

32. W. Pedrycz, P. Ekel, R. Parreiras, Fuzzy Multicriteria decision-making: Models, methods and applications, John Wiley \& Sons, Ltd. Chichester, UK (2010).

33. R.M. Rodríguez, A. Labella, L. Martínez, An overview on fuzzy modelling of complex linguistic preferences in decision making, International Journal of Computational Intelligence Systems 9 (2016) 81-94.

34. R.M. Rodríguez, L. Martínez, An analysis of symbolic linguistic computing models in decision making, International Journal of Computational Intelligence Systems 42 (2013) 121-136.

35. R. Urena, F. Chiclana, H. Fujita, E. Herrera-Viedma, Confidence-consistency driven group decision making approach with incomplete reciprocal intuitionistic preference relations, Knowledge-Based Systems 89 (2015) 86-96.

36. J.H. Wang, J. Hao, A new version of 2-tuple fuzzy linguistic representation model for computing with words, IEEE Transactions on Fuzzy Systems 14 (3) (2006) 435-445.

37. T.C. Wang, S.C. Peng, S.C. Hsu, J. Chang, An approach to group decision making based on incomplete linguistic preference relations, International Journal of Information Technology \& Decision Making 4 (01) (2005) 153-160.

38. Y.M. Wang, C. Parkan, Multiple attribute decision making based on fuzzy preference information on alternatives: Ranking and weighting, Fuzzy Sets \& Systems 153(3) (2005) 331-346.

39. J. Wu, F. Chiclana, E. Herrera-Viedma, Trust based consensus model for social network in an incomplete linguistic information context, Applied Soft Computing 35 (2015) 827-839.

40. Y.Z. Wu, C.C Li, X. Chen, Y.C. Dong, Group decision making based on linguistic distributions and hesitant assessments: Maximizing the support degree with an accuracy constraint, Information Fusion 41 (2018) 151-160.

41. Y.J. Xu, R. Patnayakuni, H.M. Wang, The ordinal consistency of a fuzzy preference relation, Information Sciences 224 (2013) 152-164.

42. T. Yang, Y. Kuo, C. Cho, A genetic algorithms simulation approach for the multi-attribute combinatorial dispatching decision problem, European Journal of Operational Research 176 (3) (2007) 1859-1873.

43. L.A. Zadeh, The concept of a linguistic variable and its applications to approximate reasoning. Part I. Information Sciences 8 (3) (1974) 199-249.

44. G.Q. Zhang, Y.C. Dong, Y.F. Xu, Consistency and consensus measures for linguistic preference relation based on distribution assessment, Information Fusion 17 (2014) 46-55.

45. H.J. Zhang, Y.C. Dong, X, Chen, The 2-rank consensus reaching model in the multigranular linguistic multiple-attribute group decision-making, IEEE Transactions on Systems, Man, and Cybernetics: Systems, doi: 10.1109/TSMC. 2017. 2694429. 
46. H.J. Zhang, Y.C. Dong, E. Herrera-Viedma, Consensus building for the heterogeneous large-scale GDM with the individual concerns and satisfactions. IEEE Transactions on Fuzzy Systems, doi: 10. 1109/TFUZZ. 2017. 2697403.

47. Z. Zhang, C.H. Guo, A method for multi-granularity uncertain linguistic group decision making with incomplete weight information, Knowledge-Based Systems 26 (2012) 111-119.

48. Z. Zhang, C.H. Guo, Consistency-based algorithms to estimate missing elements for uncertain 2-tuple linguistic preference relations, International Journal of
Computational Intelligence Systems 7 (5) (2014) 924-936.

49. Z. Zhang, C.H. Guo, L. Martínez, Managing multigranular linguistic distribution assessments in large-scale multiattribute group decision making, IEEE Transactions on Systems, Man, and Cybernetics: Systems 47(11) (2017) 3063-3076.

50. Z. Zhang, C.H. Guo, Consistency and consensus models for group decision-making with uncertain 2-tuple linguistic preference relations, International Journal of Systems Science, 47(11) (2016) 2572-2587. 